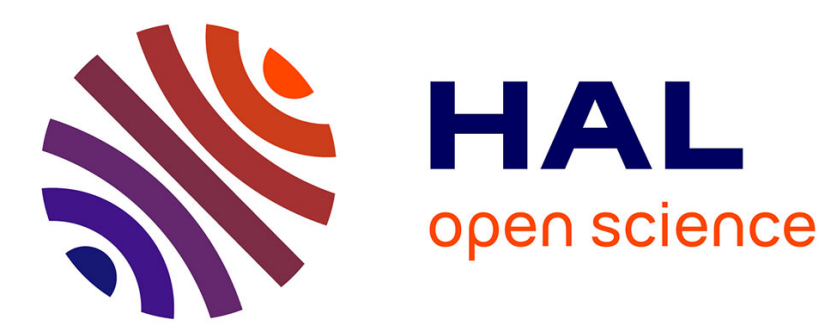

\title{
The Oscillations of the Positive Glow Corona
}

R. Sigmond

\section{- To cite this version:}

R. Sigmond. The Oscillations of the Positive Glow Corona. Journal de Physique IV Proceedings, 1997, 07 (C4), pp.C4-383-C4-395. 10.1051/jp4:1997431 . jpa-00255587

\section{HAL Id: jpa-00255587 https://hal.science/jpa-00255587}

Submitted on 1 Jan 1997

HAL is a multi-disciplinary open access archive for the deposit and dissemination of scientific research documents, whether they are published or not. The documents may come from teaching and research institutions in France or abroad, or from public or private research centers.
L'archive ouverte pluridisciplinaire HAL, est destinée au dépôt et à la diffusion de documents scientifiques de niveau recherche, publiés ou non, émanant des établissements d'enseignement et de recherche français ou étrangers, des laboratoires publics ou privés. 


\title{
The Oscillations of the Positive Glow Corona
}

\author{
R.S. Sigmond \\ The Electron and Ion Physics Group, Physics Department, Norwegian University of Science and \\ Technology, 7034 Trondheim, Norway
}

\begin{abstract}
The paper treats experiments, computer simulations, and the physical explanation of the oscillatory behaviour of positive glow coronas at or below atmospheric density. Experiments show that most positive glows have ionization regions that oscillate with frequencies depending on the gas, geometry, and average discharge current. Our circuit simulating program CIRCSIM with the general gas discharge element GPCSI reproduces quantitatively Beattie's sphere-to-plane coronas in air, and also our cylindrical coronas in air and in argon with additives. The oscillations demand a fast secondary ionization (feedback) mechanism, and are damped or inhibited by slower feedback processes like positive ion or metastable feedback, and detachment from negative ions. Linear damped oscillations are due to ionization region capacitance and equivalent inductance. Nonlinear self-sustained oscillations are formed by periodic quenching by the generated positive ions.
\end{abstract}

\section{INTRODUCTION}

This paper treats the oscillatory behaviour of positive glow corona discharges. in gases at or below atmospheric density, as studied by experiments, computer simulations, and physical reasoning. I believe that it provides a general physical answer to what has been, in my opinion, the last remaining major mystery of classical corona physics. The paper is organized as follows:

1. Introductory remarks and selected references for positive glow coronas.

2. Experimental observations of free and forced positive corona oscillations: typical recordings, and experimental indications for connections between oscillation types and discharge parameters.

3. Computer simulations: the simulation model, some important simulations of positive glow coronas, and simulation indications for connections between oscillation types and discharge parameters.

4. The physical mechanism of the positive glow oscillations: the impedances of the ionization and drift regions of coronas, passive oscillatory properties, linear and nonlinear free-running oscillations.

5. Conclusion: when and why positive glow oscillation and pulses occur.

All gases used in experiments and simulations in this paper have room temperature. The letter $g$ is used for gas density instead of the customary $n_{g}$, to save one letter in all simulation screens.

A corona discharge is a low current $(\approx \mu \mathrm{A})$ gas discharge where the electrode geometry concentrates all ionization processes in a region close to the high field corona electrode. The rest of the discharge space forms a drift region where few charges are created but where electrons, ions and excited neutrals drift and react on their way towards the other, low field electrode [1,2]. A positive glow corona has a positive corona electrode, and is characterized by an ionization region that evenly covers the high-field surface area of the electrode. In positive wire-cylinder coronas the whole wire surface is glowing, in contrast to negative Trichel type coronas, which burns in contracted, nearly evenly spaced spots along the wire. Figure 1 illustrates such a positive cylindrical corona and its commonly used external electrical supply circuit. (The other common positive corona type, the positive streamer corona, occurs when the glow ionization region produces more ions and electrons than can be conducted away to the electrode. The conducting plasma column thus formed pushes the ionization region away from the corona electrode surface and permits it to propagate into and through the drift region. This type of corona will not be further discussed here.) 


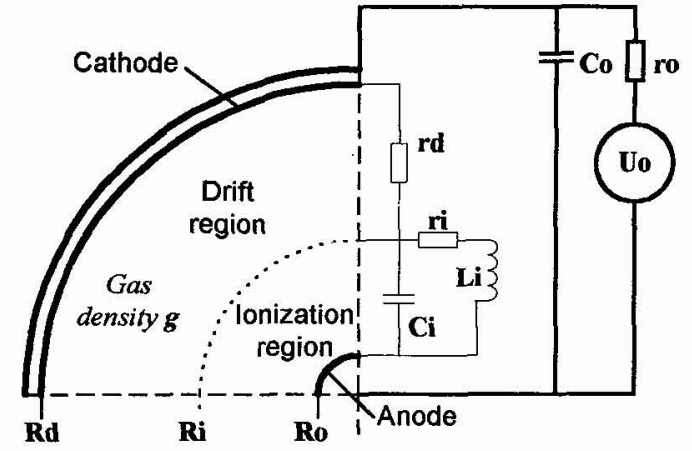

Figure 1. Schematic drawing of a quadrant of a cylindrical positive corona configuration with anode radius Ro and cathode radius Rd. Co, ro and Uo forms the real external circuit, while rd, ri, $\mathrm{Ci}$ and $\mathrm{Li}$ represent components of a simplified corona stability model, to be discussed in later sections. The ionization region is drawn abnormally large, for clarity.

As reported in the 1965 book by Loeb [3], the early investigators found positive coronas at and just above the onset voltage to consist of burst pulses and occasional weak "pre-onset" streamers. At higher voltages (and currents) these pulses either developed into streamers, or melted together on the oscilloscope screen to form the steady positive glow corona, often called the "Hermstein glow" [4] or "Uhlig ultracorona" [5].

In these early works the positive glow was generally described as "steady", with no systematic reports of oscillations. Colli et al [6] in 1954 reported on oscillatory behaviour of positive cylindrical coronas. It was, however, Beattie who in 1975 [7] pointed out that most apparently steady positive glow coronas really had violently oscillating or self-pulsed ionization regions. This pulsing is fast, in the $100 \mathrm{kHz}$ range, and does not much affect the corona surrent, which is induced mainly by the slowly

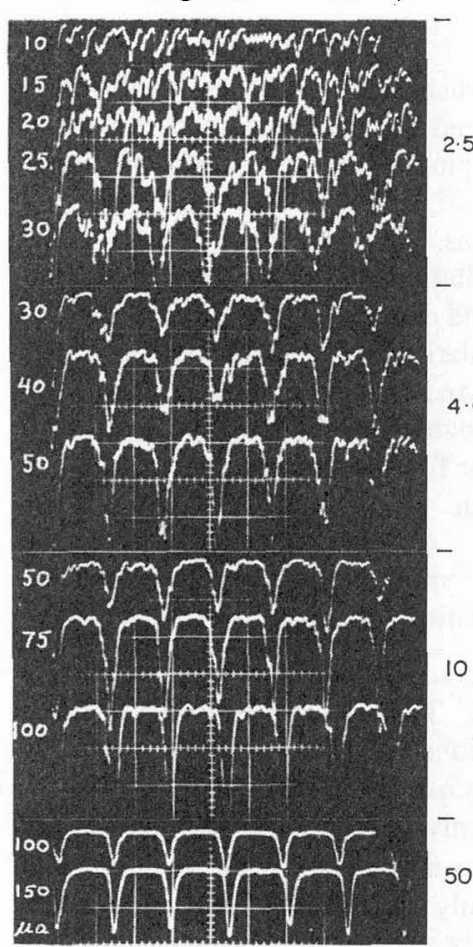
drifting positive ions. When observed by a free-running oscilloscope the pulses are easily mis-read as ordinary discharge current noise. Only by observing and triggering on the light emission from the coronas the pulses are readily visible.

Following Beattie, the positive glow oscillations were most actively studied in Trondheim, and sometimes reported upon [8-10]. We found that the oscillations were very dependent upon the corona gas composition. For example, pure argon gave no oscillations at all, but small additions of gases like $\mathrm{N}_{2}, \mathrm{CO}_{2}$, or $\mathrm{SF}_{6}$ made it oscillatory. Air in the same corona vessel gave strong oscillations, but at appreciably higher frequency than with argon as the main gas. In all cases the oscillations were practically unaffected by changes in the external electric circuit parameters. This gave us two motives for further studies: to exploit the sensitivity to gas composition in a cheap detector for trace gases, and to find the physical mechanism responsible for the oscillations. As will be told below, the latter was not at all evident.

Figure 2. Beattie's photomultiplier oscillograms of the light from a positive glow corona, at increasing values of the average discharge current. Sphere-to-plane geometry with sphere radius $16 \mathrm{~mm}$ and the plane $24 \mathrm{~mm}$ away. Gas: 1 bar of $\mathrm{O}_{2} / \mathrm{N}_{2}=10 \% / 90 \%$. Voltage around $40 \mathrm{kV}$. Time scale $5 \mu \mathrm{s}$ per major division. The average (DC) current is noted to the left of the oscillograms, while the relative light output scale is printed to the right. The pulses are completely spontaneous and free-nunning, and strongly nonlinear (no light emitted between the pulses).

\section{SOME TYPICAL EXPERIMENTAL RECORDS OF POSITIVE GLOW OSCILLATIONS}

Figure 2 shows Beattie's oscillograms [7] of the light output from the positive glow corona between a spherical anode of $16 \mathrm{~mm}$ radius and a plane cathode $24 \mathrm{~mm}$ away, in a $10 \% / 90 \% \mathrm{O}_{2} / \mathrm{N}_{2}$ mixture at 
1 bar pressure, at $\mathrm{DC}$ currents $10-150 \mu \mathrm{A}$. In this particular case the free-running pulses had a remarkably current-independent period of about $8 \mu$ s and amplitudes increasing proportionally to the current. (Note the contrast to negative corona Trichel pulses, which tend to hold the amplitude constant while their repetition frequency increases proportionally with the current). It should be mentioned that some other of Beattie's observations show a more "normal" frequency increase with the current.

Beattie ascertained that each individual ionization region pulse occurred simultaneously over the complete anode surface, i.e., it was the whole ionization region that was "turned on and off". He noted further that the pulse repetition period was considerably longer than the electron transit time and considerably shorter than the ion transit time between anode and cathode, but coincided well with the positive ion transit time across the ionization region. Beattie also found that some electronegative gas

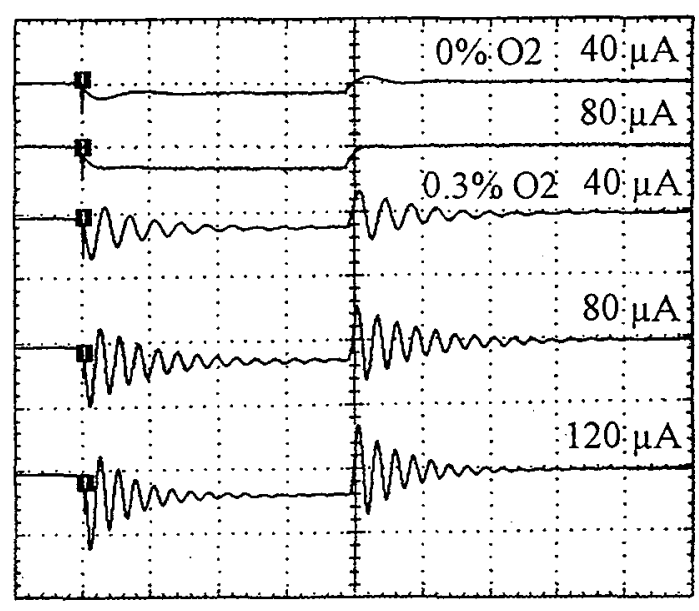
constituent seemed to be needed for oscillations to occur, and concluded that the oscillations had to be due to photodetachment from negative ions arriving in front of the ionization region. This conclusion was disputed by me [1] because the relevant cross sections seemed far too small.

Figure 3. Voltage pulse excited current oscillations in cylindrical positive glow coronas in 1/4 bar argon and argon with $0.3 \%$ oxygen for different average (DC) corona currents (values typed above the curves) [11]. Anode radius $\mathrm{Ro}=0.8 \mathrm{~mm}$, cathode $\mathrm{Rd}=13 \mathrm{~mm}$, guard-ring shielded cylinder length $80 \mathrm{~mm}$. Excitation pulse to the cathode $10 \mathrm{~V} 200 \mu \mathrm{s}$ negative. Time scale $50 \mu \mathrm{s} / \mathrm{div}$, current scale $10 \mu \mathrm{A} / \mathrm{div}$. The current is negative. Traces are averaged over about 100 sweeps.

The experimental work in Trondheim has covered both point-to-plane coronas in $\mathrm{N}_{2}-\mathrm{O}_{2}$ mixtures [10], and concentric cylinder coronas in argon with additives and in air. The cylindrical coronas had guard sections to eliminate end effects, and are directly comparable with one-dimensional calculations, see Section 3 below.

Figure 3 shows the effect of corona current variation for pure argon and for $\mathrm{Ar}+0.3 \% \mathrm{O}_{2}$, while in Figure 4 the gas composition is varied at a constant $50 \mu \mathrm{A}$ corona current [11].

Figure 4. Voltage pulse excited current oscillations in cylindrical positive glow coronas in 1/4 bar argon with different trace gases added [11] (concentrations typed above the curves), all at $50 \mu \mathrm{A}$ average corona current. Scales and other data as in Figure 3. 
The excitation is a $10 \mathrm{~V} 200 \mu \mathrm{s}$ negative pulse applied to the outer (cathode) cylinder, which ensures that the unavoidable capacitive current pulse has the opposite polarity of an absolute increase in the (negative) discharge current.

Notable features of the Figures 3 and 4 and of all our other similar experiments are:

- Pure Ar shows a nearly pure $\mathrm{RC}$ relaxation response, without oscillatory tendency.

- $0.01 \%$ of the highly electronegative $\mathrm{SF}_{6}$ in $\mathrm{Ar}$ has the same effect as $0.05 \%$ of the correspondingly less electronegative $\mathrm{O}_{2}$, - but also the same effect as $0.01 \%$ of the very weakly electronegative $\mathrm{CO}_{2}$.

- Hydrogen additives also induce oscillatory behaviour, but $\mathrm{D}_{2}$ gives lower frequencies than $\mathrm{H}_{2}$.

- An increase of additive concentration increases the oscillation frequency.

- At higher concentrations of $\mathrm{O}_{2}, \mathrm{SF}_{6}$, and $\mathrm{CO}_{2}$ the oscillations become free, and then become nonlinear.

- For all linear oscillations in a given gas mixture, the frequency increases proportionally to the square root of the DC discharge current: $f \propto \sqrt{I}$.

Please note that any changes in current or gas composition also entail changes in the corona voltage, with corresponding changes in electron and ion transit times and discharge reaction times.

\section{COMPUTER SIMULATIONS OF POSITIVE GLOW CORONAS}

The computer simulations form an all-important part of the present study. The simulation engine is quite unique, and to judge its conclusions, it is essential that the.reader know its basic principles, its strenghts and its weaknesses. It differs from more conventional computer models of gas discharges by:

- treating discharges as elements in a general electrical circuit simulation program

- handling small numbers of charged particles as Poisson distributed integers

- handling drift by shuffling particles as bunches from one computing cell to another without splitting.

\subsection{Gas discharges and the CIRCSIM circuit simulation system}

A given corona discharge may have concentrated regions containing millions of electrons and ions in non-linear Coulomb interaction, and other regions where one single electron is the seed that spawns new millions elsewhere. Our gas discharge simulation model GPCS1 [12] handles both the continuum aspects of the many as well as the stochastic integer nature of the few. The external electrical circuit feeding the discharge is often essential to its stability and mode, and is therefore included in the modeling.

GPCS1 forms one special circuit element in the general-purpose electrical circuit simulation program CIRCSIM developed by me and my students for PC type microcomputers. The CIRCSIM algorithm calculates the circuit voltages and currents by intelligent trial-and-error adjustments of all node voltages until the Kirchhoff current law is satisfied for all nodes to a specified accuracy. It solves no explicit matrices and does no linearizations at all. Circuits may be built from one or more discharge elements combined with all common passive and active electronic circuit components, like R, C, L, thermistors, diodes, Zeners, transistors, transformers, transmission lines, operational amplifiers, and voltage and current waveform supplies.

At every time step $k$, the main CIRCSIM algorithm gives each circuit element $i$ its input voltages $V_{i, k}$. On the basis of these, and previous voltages $V_{i, k-l}$ and currents $I_{i, k-l}$ stored by the elements, each element is required to give back the present currents $I_{i, k}$. These are then used by the CIRCSIM algorithm to calculate the deviations from Kirchhoff's current law for each node, and to estimate the next iterative set of node voltages within the same time step.

\subsection{The GPCS1 gas discharge element}

\subsubsection{Geometries, space and time subdivisions, inputs and outputs}

The GPCS1 element has a selectable geometry with one space coordinate. GP1 has plane parallel electrodes, GCl has concentric cylinders, and GS1 has concentric spheres. The discharge volume is subdivided along the space coordinate $R$ into 50 or 120 computing shells of widths inversely proportional 
to the Laplacian electric field. For each CIRCSIM time step $d T i$ the electrons, negative ions, positive ions, and metastables are moved between these shells according to the local electric field, mobilities and diffusion coefficients; and excite, ionize, attach, and detach. The electric field $E$ is recalculated for each time step by the Poisson equation. The electrode current is calculated by the Shockley-Ramo theorem and added to the current due to the electrode capacitance, to give the element output current.

To speed up the simulation, the GPCS1 element may change the CIRCSIM time step $d T i$ according to the speed of the discharge processes. Also, it may internally subdivide $d T i$ into DivT local time steps $d T e$, usually 10-100. Inside each CIRCSIM time step $d T i$ only the electrons are moved and react, while heavy particles and imposed voltages are frozen. Interaction with the external circuit can only take place each main time step $d T i$.

The discharge elements have two main circuit terminals A and B for connection to the normal electrical supply circuit. In addition they have particle input and output (signal) terminals. The particle input terminals accept voltages as signals, equal to currents of electrons or ions to be liberated from the electrodes by external means (like photoelectrons). By connecting these terminals to CIRCSIM voltage waveform generators, any combination of ion and electron pulses can be liberated from the electrodes. Likewise, electrons, ions and metastables arriving at a discharge electrode may be selected to generate output signal voltages equal to the particle currents (in equivalent amperes, even for neutrals).

The GPCS1 circuit element, inserted into a typical voltage supply and current recording circuit, is illustrated in the Figure 5 below.

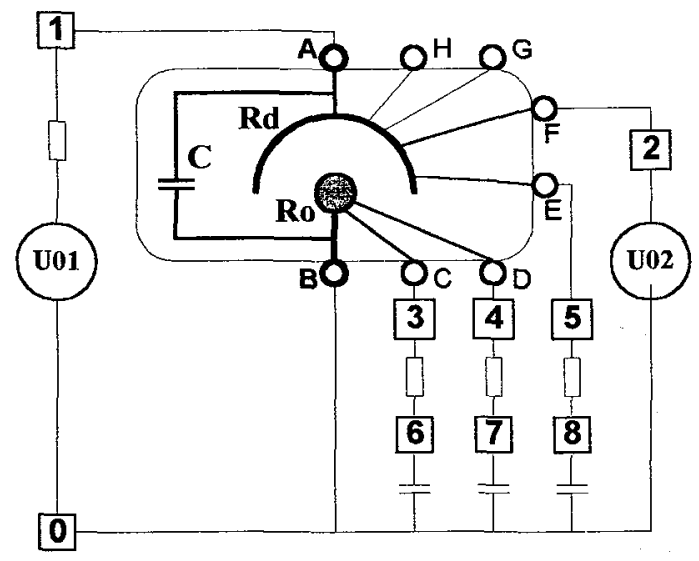

Figure 5. The CIRCSIM GPCS1 plane/cylindrical/spherical discharge element, inserted in a typical voltage supply and current recording circuit.

The anode radius is $\mathrm{Ro}$, the cathode radius $\mathrm{Rd}$, and the gap capacitance $C$. U01 is the applied voltage with internal resistance $\mathrm{r01}$, while $\mathrm{U} 02$ injects an electron current from the cathode. $\mathbf{A}$ and $\mathbf{B}$ are the real corona current terminals. C, $D$, and $E$ give out signal voltages numerically equal to the currents of electrons and negative and positive ions hitting the electrodes, while nodes 6,7 , and 8 give out the corresponding RC-filtered signals. $F$ is a signal terminal used for injecting an electron current from the cathode. $\mathrm{G}$ and $\mathrm{H}$ are signal terminals which can be used for particle input or output, but which are not used in the present circuit.

\subsubsection{The charged particle drift algorithm}

The charged particle drift algorithm is the main engine inside the GPCS1 discharge model. The plane, cylindrical, and spherical electrode geometries are all mathematically one-dimensional, i.e., the discharges develop along one space coordinate only. This coordinate is called $R$, or $R k$ when we refer to the $R$ of the space sheil no. $k$. The inner electrode is at $R=R 0$, and the outer electrode at $R=R d$. The discharge volume is subdivided into $T M$ shells, $k=I . T M$, where at present $T M=50$ or 120 . The shells are represented by elements in the TrArrays (for Transit Arrays). Each type of charged particles uses two array rows, i.e., two array elements per space shell, while neutral diffusing particles use one.

The drift of any charged particle type $i$ is simulated by retaining the particles in each half-shell for half the time needed to cross the shell, and then transferring all those particles to the next half-shell. Thus, by this two-step drift algorithm the particles move in bunches which may be added, but never split up. The use of two array elements (half-shells) per shell is necessary in order to avoid particle runaway in case of slowly decreasing particle velocity downflow. When below a selected number, the content of any cell is treated as a stochastic integer. In this way unphysical fractional particles are never created, and the ordinary numeric diffusion effect is avoided. The two-step drift algorithm has two drawbacks, however: a certain scatter in the charged particle transit time, and comparatively large particle current fluctuations (numerical noise) due to the creation of occasional empty shells. 


\subsubsection{Particle diffusion and reactions}

Particle diffusion and reactions (like ionization and attachment) are computed separately from the drift. The algorithm scans through the TrArrays and calculates the reactions in each cell from the reaction rate functions (see below), and then simulates diffusion by three-point averaging weighed according to the diffusion coefficient to be simulated.

All reactions that may create particles in critical feedback positions, like electrode processes, detachment, and photoionization are sent through a random Poisson integer distribution routine if the particles created in one step are few. Thus, fractional electrons are avoided where they could have caused numerically important but completely unphysical avalanches.

\subsubsection{The current induced in the external circuit}

The current in the external circuit is due to two sources: the current induced by the moving particles, and the current due to the capacitance of the gap. CIRCSIM treats the latter as an ordinary capacitor.

The electrode current $I_{q k}$ induced by a charge $q$ moving with a radial velocity $v$ in a shell number $k$ at position $R k$ is given by the Shockley-Ramo theorem:

$$
I_{q k}=q v E_{L k}^{I} \text {, where } E_{L k}^{I} \text { is the Laplacian field at } R k \text { due to } 1 \mathrm{~V} \text { across the gap. }
$$

The total charge-induced current can be found simply by summing this equation over all charges moving in all shells. This may be called the charge drift method.

A mathematically equivalent and much more elegant method is found by taking the partial derivative of the charge drift method formula. The resulting charge derivative method will only contain a sum over the changes in the net shell charges from time step to time step, and computes appreciably faster. However, in GPCS1 it gives too much noise to be used, because of the two-step charged particle drift algorithm with infrequent transfers of particles from cell to cell.

\subsubsection{Basic physical processes included in the GPCSI}

The GPCS1 handles the basic discharge processes as follows:

- Transport equilibrium is assumed for all processes

- Mobilities and diffusion coefficients are field independent

- No-barrier interaction processes are always exothermic, and will have maximum and fieldindependent reaction rạtes at low fields, falling off at higher fields. Three-body electron attachment in air is of this type. Reaction rates from literature are used.

- Barrier processes, like electron impact ionization and dissociative attachment, need a certain energy to occur. For these reaction rates GPCS1 uses Townsend-type formulas:

$$
\frac{\varphi}{g}=v \cdot \frac{A}{g} \cdot \exp \left(-\frac{B / g}{E / g}\right) \quad v: \text { drift velocity, } E: \text { el. field, } g: \text { gas density, } A / g \text { and } B / g \text { : constants. }
$$

\subsubsection{Comparison with other, more conventional models}

The CIRCSIM / GPCS1 model has proven to be very flexible, stable and reliable. It handles all types of external circuits, and all types of applied voltages: DC, AC HF, pulsed. At high plasma densities it may develop a fatal instability, probably because the plasma frequency gets too high in relation to the time step. This, however, never happens in coronas. Its main weakness derives from its main strength: it simulates discharges so well that it also simulates the discharge noise. In experiments using digital oscilloscopes, like Figures 3 and 4, one has to remove the noise by averaging over 20-200 sweeps. At a simulation speed of one millisecond per day such noise reduction by averaging is prohibitively slow.

Other, more conventional discharge simulation models, like the corona computations of Morrow [13], use continuous mathematics and often more implicit algorithms. They usually allow avalanche multiplication and feedback of fractional electrons and ions, and must be reprogrammed for each change of the external circuit, if included at all. These models are usually faster and have much less noise than GPCS1. Thus, the most efficient simulation strategy is probably to use CIRCSIM / GPCS1 for initial 
surveys, external circuit changes, varying applied voltage types, and for checking continuous models; and to use the checked continuous models for series runs.

\subsection{Selected GPCS1 simulations of positive coronas}

The GPCS1 discharge model has about 40 adjustable parameters representing gas discharge process parameters. The strategy for searching for sets of parameters giving simulations which fitted experiments was to let well-published coefficients (like ionization and attachment coefficients, drift velocities, and diffusion coefficients) keep their values, select some few (like detachment, secondary ionization coefficients, and photoionization) for systematic variation, and put the rest equal to zero. The main experimental features to be reproduced were current-voltage characteristics, current-frequency curves, and oscillation damping. The systems simulated were our concentric cylinder corona chamber (data in Figure 6) filled with argon+additives or air at $1 / 4,1 / 8$, and $1 / 16$ bar, and Beattie's sphere-plane system (data in Figure 8). The same set of discharge parameters for a given gas had to fit at all pressures.

The usual procedure was to adjust the sum of the secondary ionization and photoionization coefficients to make the current-voltage characteristics fit quantitatively over the pressure range, and then adjust the individual components of this sum to make the oscillation characteristics fit as well.

In this way the positive corona discharge systems described in Section 2 has been simulated running two $200 \mathrm{MHz}$ Pentium PCs for more than one year. Even at a simulation speed of about one day per millisecond the number of runs recorded is large, and only the most important ones may be displayed here.

Figure 6 shows simulations of the initiation of a positive corona in the cylindrical corona chamber used for Figures 3 and 4, done both with the 50 shell and the 120 shell models. The agreement between the models is satisfactory, and the 50 shell model has been used for nearly all further simulations.
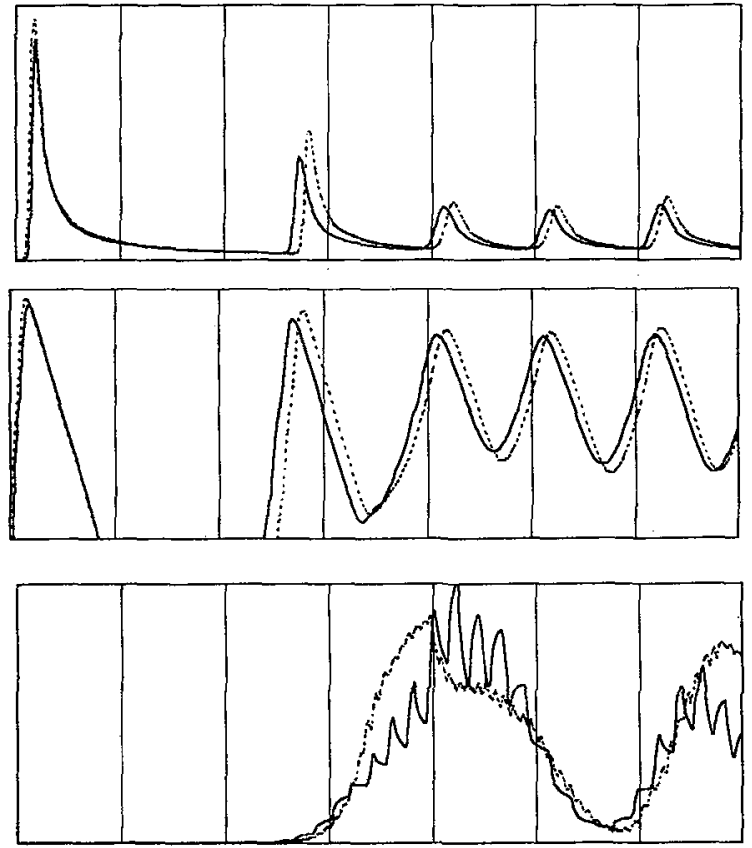

Corona current, linear scale $0-1800 \mu \mathrm{A}$

Solid curves 50 shell, dotted curves 120 shell models. The corona current is computed from the ShockleyRamo theorem, and is due to all charges moving in the corona gap between the inner anode at $R o=0.8 \mathrm{~mm}$ and the outer cathode at $\mathrm{Rd}=13 \mathrm{~mm}$. The length of the corona section is $80 \mathrm{~mm}$.

Electron current arriving at the anode, logarithmic scale $10^{-8}-10^{-2}$ A (6 decades!). The negative ions arriving at the anode follows the same curve, one decade lower.

With time the corona current and the electron current to the anode settle into regular, highly nonlinear oscillations of period about $11 \mu \mathrm{s}$, against observed about $3 \mu$ s. This is due to the photon feedback to the cathode. Photoionization gives the observed period.

Positive ion current arriving at the cathode, linear scale $0-400 \mu \mathrm{A}$. The first positive ion cloud inhibits ionization for about $30 \mu \mathrm{s}$, and then the second, smaller peak follows. With time the positive ion flow gets stationary. The ripple on the $\mathbf{5 0}$ shell curve is computational noise due to the special drift algorithm.

Time scale $0-70 \mu \mathrm{s}$, common for all curves.

Figure 6. GCl simulation of cylindrical corona in 1/4 bar "air", with ionization and attachment, but without detachment and photoionization. The secondary processes included are about $99 \%$ photon and $1 \%$ positive ion feedback to the cathode.

Figures $7 \mathrm{a}, \mathrm{b}$ present computer simulations for the same cylindrical corona with respectively photon feedback to the cathode (a) and to the gas (photoionization, b), and with a stepwise increasing electron current injected from the cathode. In both cases the externally injected electron current damped the 
oscillations, but only case b), with photoionization as the main feedback mechanism, gave current-voltage and oscillation characteristics in quantitative agreement with our air corona measurements. In other simulations the discharge current was varied. All linear oscillations followed the $f \propto \sqrt{I}$ law.

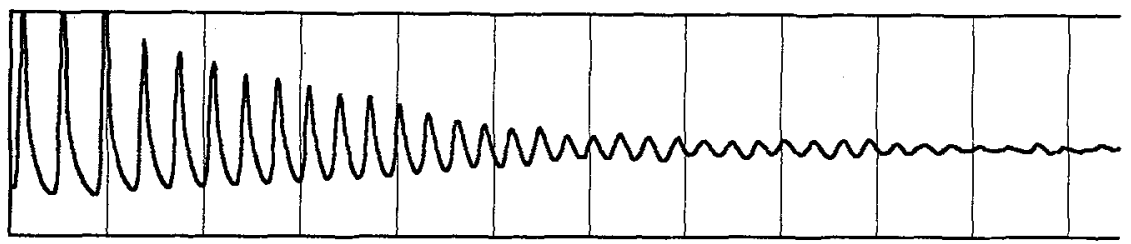

Corona current $0-400 \mu$ A linear. Photon feedback to the cathode, no photoionization.

Time $25 \mu \mathrm{s} / \mathrm{div}$.
a) $0.1 \quad 2.1$
$4.1 \quad 6.1$
$8.1 \quad 10.1$
$12.1 \quad 14.1 \quad 16.1$
18.1
20.1 nA electron current injected.
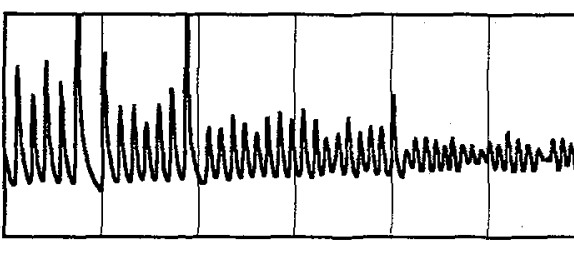

b) $0.1 \quad 1$.

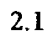

3.1

4.1

5.1

6.1

7.1

8.1

9.1

Corona current 0-250 $\mu \mathrm{A}$ linear. Photoionization, photon range $\approx 1 \mathrm{~mm}$. No photon feedback to the cathode.

Time $25 \mu \mathrm{s} / \mathrm{div}$.

Figure 7 a-b. GCl simulation of cylindrical corona in 1/4 bar "air", with ionization and attachment, but without detachment. In both cases a steady injected electron current quenches the oscillations. Case b) has oscillation period in agreement with exp.

Similar simulations were made of the cylindrical corona chamber filled with argon plus additives, using a suitably pulsed voltage source. For pure argon a strong metastable feedback to the cathode had to be introduced to get agreement with experimental current-voltage characteristics and absence of oscillations. Some photon feedback to the cathode was also included, but played no role for pure argon. Additive gases were successfully simulated by quenching the metastable population while retaining the photon feedback to the cathode. Photoionization gave too short oscillation periods for the argon-based mixtures. Again all linear oscillations followed the $f \propto \sqrt{I}$ law.

Finally, attempts were made to simulate the Beattie corona shown in Figure 2 above, using the GS1 spherical geometry model. The $16 \mathrm{~mm}$ radius spherical anode was simulated by a sphere, but the plane cathode $24 \mathrm{~mm}$ away had to be simulated by a second concentric sphere of $40 \mathrm{~mm}$ radius. These simulations used a slowly rising voltage ramp, in order to find parameter values that gave approximately current independent oscillation frequencies. Such oscillations necessarily had to be strongly non-linear, to escape from the $f \propto \sqrt{I}$ law. The quite successful result is shown in Figure 8 .

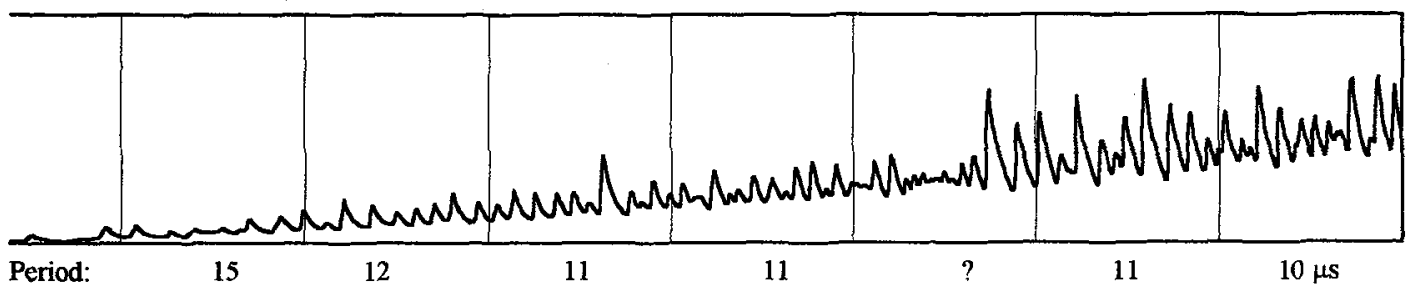

Figure 8. GS1 simulations of the Beattie corona of Figure 2 using concentric spheres of 16 and 40 mm radius, and ionization and attachment corresponding to 1 bar of $\mathrm{O}_{2} / \mathrm{N}_{2}=10 \% / 90 \%$, no detachment, and photoionization of the same specific range as for the other air corona simulations. Supply voltage linearly rising from 45 to $58 \mathrm{kV}$. Time scale $100 \mu \mathrm{s} / \mathrm{div}$, corona current scale $0-10 \mathrm{~mA}$. The approximate oscillation period is shown under the graph, along the time axis. 


\subsection{Main results from the computer simulations}

1) The most important conclusion from the simulations is that Beattie's and our own positive corona measurements can be simulated quantitatively using consistent and reasonable sets of discharge parameters, and without postulating unproven or controversial discharge processes.

2) The old accepted sets of primary ionization and attachment coefficients for both air [14] and argon [15] could be used without modification. Detachment in air, which has always been a subject of debate, had to be put close to zero, see below. In pure argon the metastable feedback coefficient had to be chosen so large that the corona onset occurred at an avalanche multiplication as low as 2 .

3) Positive glow oscillations occurred whenever the main secondary (feedback) processes in the discharge were fast, i.e., had avalanche generation time distance $T g$ appreciably less than the positive ion transit time across the ionization region $T p i$.

4) The oscillation or pulse period was roughly proportional to $T g$, and usually of the order of Tpi. Linear oscillations (usually pulse excited) always had frequencies following the $f \propto \sqrt{I}$ law. Non-linear pulses had longer periods, which usually were close to the positive ion transit time across the ionization region $T p i$.

5) Positive glow oscillations were damped or completely eliminated by all slow electron feedback processes, i.e., processes that gave secondary electrons with delay greater than Tpi. Processes like constant electron injection (Figures $7 \mathrm{a}-\mathrm{b}$ ), metastable feedback, positive ion feedback, and electron detachment from negative ions, all inhibited positive glow oscillations.

6) For the air coronas, the main feedback process had to be photoionization, to make $\mathrm{Tg}$ small enough to reproduce the high experimental oscillation frequencies.

7) For argon, feedback by metastables had to be the main secondary ionization process, with a small amount of photon feedback to the cathode added. Additives caused oscillations by quenching the metastable feedback, thus increasing the importance of the remaining photon feedback. Photoionization would give oscillation periods 3 times shorter than observed, and could not be a main secondary process in the argon based coronas.

8) Linear, pulse excited oscillations did not always readily transform into free-running nonlinear pulses, even when the parameters should encourage this. Often a larger pulse shock had to be given to start free-running pulses. (The computer, like me, could not always find any reason for positive feedback in the linear regime!)

\section{THE PHYSICAL MECHANISM OF THE POSITIVE GLOW OSCILLATIONS}

There are three different features of positive glow coronas that must be explained: the passive oscillatory response to a disturbance, the negative damping that in some cases makes free-running linear (sinusoidal) oscillations, and the completely nonlinear glow pulses. The third is simple, see Section 4.6., but the first two require discussion of the small signal equivalent circuit parameters of the corona regions.

\subsection{The effects of a positive space charge shell. The ionization region capacitance}

To discuss the oscillatory properties of the positive corona, we need the real and equivalent circuits drawn in Figure 1, as well as two new figures 9 and 10 which illustrate the effects of a shell of positive space charge on the field distribution and on the ionization integral of a low-current positive glow corona. The spherical simulated Beattie corona of Figure 8 has been chosen for convenience, but our cylindrical corona geometries show the same general features.

Figure 9 shows that a shell of positive charge $Q$ around a positive inner electrode always will diminish the field inside and increase the field outside the charge shell. The net effect of $Q$ on the ionization integral $I i=\int_{R o}^{R d}(\alpha-\eta) d R$ will always be detrimental, as long as the curve of the effective ionization coefficient $\alpha^{\prime}=\alpha-\eta$ vs. the reduced field $E / g$ has a positive curvature. This is the case for all coronas treated in this paper. For the Beattie corona model of Figures 8-10 the ionization region limit, where $\alpha^{\prime}=0$, is at $R i=22 \mathrm{~mm}$, so a $Q$ at this position certainly will diminish $I i$. However, the important point is that this does not make a negative, resistive $180^{\circ}$ feedback, but a capacitive $90^{\circ}$ feedback. 
The proof is that a current increase $\Delta I$ does not give a proportional reduction $\Delta V q$ of the ionization region voltage, while a charge increase $\Delta Q=\int \Delta I d t$ does. The capacitance $C q=\Delta Q / \Delta V q$ at $R q$ is easily calculated, and equals $12 \mathrm{pF}$ for $R q=R i=22 \mathrm{~mm}$ at the edge of the ionization region in the Beattie corona model of Figs.8-10. This identifies the ionization region equivalent capacitance $C i$ in Figure 1 .
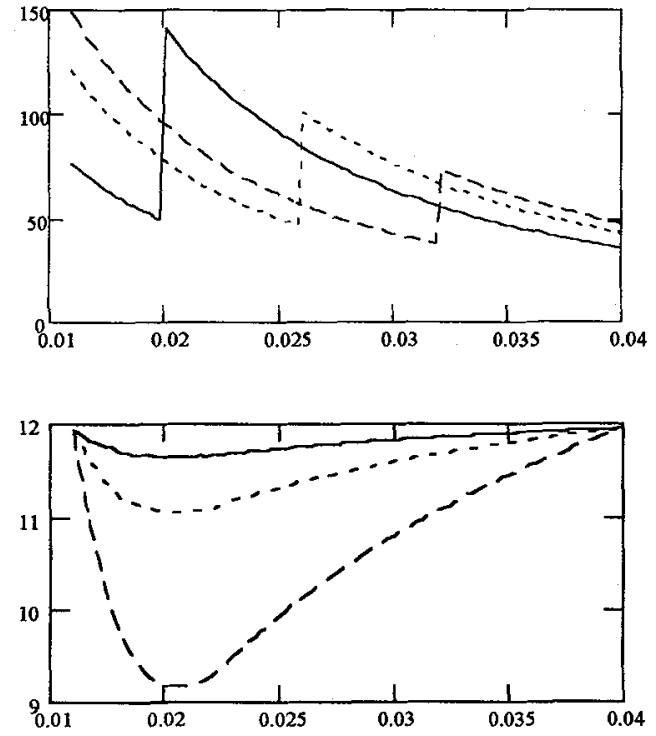

Figure 9. The effect of a given spherical, positive shell charge $Q$ on the reduced electric field $E / g$ distribution between the anode and the cathode spheres in the GS1 approximation to the Beattie geometry (see Figure 8 legend), as function of its position $R q$. Anode radius $16 \mathrm{~mm}$, cathode radius $40 \mathrm{~mm}$, $40 \mathrm{kV}$ gap voltage, and $E / g$ scale $0-150 \mathrm{Td}$. No other space charges are present.

$Q$ will always diminish the field inside it and increase it outside. Its effect diminishes with the distance from the anode. The curve for $Q=0$ (not drawn, for clarity) goes mid-between the right part of the solid curve and the left part of the dashed.

The ionization region limit, where $(\alpha-\eta)=0$, is at $R=22 \mathrm{~mm}$.

Figure 10. The effect of a shell charge $Q$ on the ionization integral $I i=\int_{R o}^{R d}(\alpha-\eta) d R$, as function of the shell charge position $R q$, for three values of $Q$, in the GSI approximation to the Beattie geometry. Anode radius $16 \mathrm{~mm}$, cathode radius $40 \mathrm{~mm}, 40 \mathrm{kV}$ gap voltage. No other space charges are present. Note that the effect of a positively charged shell will always be detrimental for the corona gaps of this paper. Only for much smaller inner electrode radii will the ionization integral show a weak maximum for $Q$ positions close to the inner electrode.

\subsection{The drift region equivalent resistance}

A resistive effect $r d$ of the drift region is defined by the drift region's immediate current change $\Delta I$ when the drift region voltage is changed by $\Delta V d, r d=\Delta V d / \Delta I$. At any given moment the corona drift region will contain numbers of positive and negative charges that are roughly proportional to the corona average current $I o$. As no ionizations take place here, the only immediate effect of a voltage change $\Delta V d$ is to increase the charge particle speeds proportionally. The $N i$ positive ions dominate the drift region, and we can thus write: $\quad \Delta I \approx N_{i} \mu_{i} \Delta V d \propto I o \Delta V d \quad r d \propto 1 / I o$

This allows an estimate of the drift region equivalent resistance $r d$ in Figure 1 and its current dependence.

\subsection{The ionization region equivalent inductance and resistance}

To show oscillatory behaviour, any electrical system must have two internal elements with oppositephase current-voltage characteristics (like capacitance and inductance). For the cases of a parallel-plate DC Townsend discharge or a corona ionization region, the first of these required circuit elements is the capacitance $C i$ of the region, see Section 4.1. For a short time this capacitance is able to sustain a discharge current different from the imposed DC current. The voltage will lag behind any current change.

The second required element for oscillations is the equivalent inductance $L i$ of the discharge or ionization region. This inductance is due to the delay between the application of a voltage step to the discharge DC voltage and the subsequent discharge current change. Any DC discharge burns with a reproduction factor $\mu=1$; i.e., each electron that leaves the cathode will on the average be replaced by exactly one new electron in the next avalanche generation. A voltage step $\Delta V i$ added to the $\mathrm{DC}$ sustaining voltage will increase the reproduction factor from 1 to $1+\Delta \mu$, which will make each new avalanche generation $1+\Delta \mu$ larger than the preceding one. If the mean time between the avalanche generations is $T g$ (equal to the electron transit time in case of photon feedback alone), then the current will change with time as: 


$$
I_{i}(t)=I_{i 0} \exp \left(\frac{\Delta \mu}{T_{g}} t\right) \quad\left(\frac{d I_{i}}{d t}\right)_{0}=I_{i 0} \frac{\Delta \mu}{T_{g}}=\frac{I_{i 0}}{T_{g}} \frac{\partial \mu}{\partial V_{i}} \Delta V_{i}
$$

If we, for comparison, take a real coil of inductance $L$ and impose a voltage step $\Delta V i$ across it, then a linear current change will result:

$$
\frac{d I}{d t}=\frac{\Delta V_{i}}{L}
$$

Thus, for small voltage deviations from the DC sustainment voltage, the discharge or ionization region will act as inductances:

$$
L_{i}=\frac{T_{g}}{I_{i 0} \partial \mu / \partial V_{i}}
$$

Note that $L i$ is proportional to $T_{g}$ and inversely proportional to the DC sustainment current Iio.

Will the Townsend discharge or corona ionization region also have an equivalent resistance $r i$, meaning that a change of current $\Delta I i$ will cause a fairly immediate change $\Delta V i$ in burning voltage? Yes, one insignificant part due to the increase of charge velocities caused by field increase, and one important part due to the change of the secondary ionization processes caused by space charge distortion of the field in the region. The space charges will usually increase the ionization efficiency in a parallel plate Townsend discharge, and this makes $r i$ negative. For the ionization region in a positive corona, the effect depends on the types of secondary processes. From Figure 9 it is seen that a positive charge shell will increase the cathode field, and thus photon feedback to the cathode, while it might easily reduce the photoionization feedback to the gas near the anode. Thus the sign of $r i$ for a positive corona depends on a quantitative balance between several discharge processes, and is not at all obvious.

\subsection{The passive oscillatory properties of the positive glow corona}

If we at first neglect the equivalent resistance effects in Figure 1 by letting $r d$ be very large and $r i$ very small, then the small signal characteristics of the Townsend discharge and the corona ionization region may be modeled by the gap or region capacitance $C i$ in parallel with the discharge inductance $L i$. A voltage pulse added to the discharge sustainment voltage will then induce a train of current oscillations with the angular frequency $\omega x$ :

$$
\omega_{i}=\frac{1}{\sqrt{L_{i} C_{i}}} \propto \sqrt{I_{i 0} / T_{8}}
$$

This $\sqrt{I_{i 0}}$ frequency dependence has previously been observed in plane parallel Townsend discharges [16] and in positive concentric cylinder coronas [6], and is a main feature of all linear oscillations observed in the present experiments and simulations.

Please note from eq.(7) that the equivalent inductance $L i$ also is proportional to the effective avalanche generation duration $\mathrm{Tg}$, and inversely proportional to the sensitivity of the reproduction factor to changes in the ionization region voltage $\partial \mu / \partial V$. The oscillation period is not determined by the positive ion transit time across the ionization region (but oscillations with longer periods become highly damped, see below). For linear oscillations the positive ions will affect $r i$ but not $\boldsymbol{\alpha} i$.

It is immaterial whether the oscillations are induced by applied pulses or by the natural stochastic fluctuations of the discharge current. In all cases a resistive impedance ro in the external circuit will damp these oscillations, as will the effective equivalent resistance $r d$ of a corona drift region.

\subsection{Linear free-running positive glow oscillations?}

In no way may the (positive) series impedances $r o$ and $r d$ make the oscillations self-sustained. For this, a negative effective resistance must exist. In a plane parallel Townsend discharge or in the ionization region of a negative corona, such a negative resistance may be provided-by the space charge field of the positive ions, which will increase the cathode field and thus the efficiency of the secondary cathode processes. This will reduce the voltage necessary to sustain the discharge, and the $V i(I i)$ curve will get a 
negative slope. In the ionization region of a positive corona, on the other hand, Section 4.4. concluded that the sign of $r i$ is more difficult to predict.

We now can discuss the complete small-signal equivalent network for a corona discharge shown in Figure 1. For a parallel-plate Townsend discharge the value of $r i$ is readily measured, being the slope of the $V(I)$ curve at small $(\mu \mathrm{A})$ currents. For a corona ionization region, $r i$ will usually be masked by the drift region impedance $r d$, since it is the sum $r i+r d$ that is accessible as the slope of the $V(I)$ characteristic of the corona. Only when $r i$ is negative and $r d+r o$ sufficiently large will $r i$ reveal itself, not by the slope but by making the current oscillatory.

It is easily shown [16] that the equivalent circuit of Figure 1 has two possibilities of instability. Both demand negative $r i$ :

$$
\begin{array}{lll}
\text { Catastrophic: } & r_{i}+r_{d}+r_{0}<0 & \text { (when } C o \text { is some picofarads only), leading to spark. } \\
\text { If } C o \text { is much larger: } & r_{i}+r_{d}<0 & \text { leading to recurrent spark discharging of } C o \text {. } \\
\text { Oscillatory: } & r_{d}>L_{i} / C_{i}\left|r_{i}\right| & \text { for C0 greater than some ten picofarads. }
\end{array}
$$

A high drift region resistance, i.e., a low corona current, should encourage growing linear oscillations. However, the sign of $r i$ may change from one positive corona to another, as born out by the reluctance of our simulations to give growing oscillations in the linear, small signal range. This is radically different from negative coronas, where the positive ions formed by a first avalanche obviously increases the cathode field and all secondary processes, making $r i$ so negative that the current growth becomes exponential even in the small-current range. Trichel pulses have no growth inhibitions.

\subsection{The nonlinear positive glow pulses}

In contrast to linear positive glow oscillations, these are easy to predict and explain. Let us assume, in Figure 10, that the corona has such secondary (feedback) processes that the corona on the average needs an ionization integral $I i=11$ to burn with a reproduction factor $\mu=1$. We start the reasoning and the corona without any space charge, with an electron at the edge of the ionization region at $R=22 \mathrm{~mm}$. This makes an avalanche on its way to the anode. As then $l i=12$ and $\mu>1$ the ionization grows, and if the feedback processes are fast enough a substantial positive charge $Q$ will have time to form before $I i$ falls below 11 (look at the $I i$ curve for the largest $Q$ ). After $Q$ has reached this point it continues to drift outwards, and $\mu$ falls significantly below 1 . All ionization ceases, and in simulations the corona dies out if not some slow feedback also exists to start the next pulse. A new pulse then starts when the $Q$ has drifted out far enough to again make $I>11$, i.e., $\mu>1$.

\section{CONCLUSIONS}

The oscillatory behaviour of positive glow corona discharges in gases at or below atmospheric density has been investigated using experiments, computer simulations, and physical reasoning. The combined results should provide a general answer to this last remaining major problem of classical corona physics:

1) The positive glow corona gets oscillatory behaviour when the dominating secondary feedback processes of the corona are rast compared to the transit time of positive ions across the ionization region. A minute amount of slow feedback or external electron generation may be necessary continuously to provide initiatory electrons in the case of nonlinearly self-pulsed coronas.

2) It is immaterial whether the feedback is photoelectron emission from the cathode or photoionization of the gas outside the ionization region, as long as the feedback fulfills the speed criterion 1). The linear oscillation periods may thus be much shorter than the positive ion transit time across the ionization region, but not much longer because of condition 1).

3) Linear positive corona oscillations generally have oscillation frequencies proportional to the square root of the average corona current. Nonlinear repetitive positive glow pulses temporarily quench all ionization (see point 6), resulting in lower and less current dependent repetition rates.

4) Slow secondary processes, like positive ion or metastable feedback, or detachment from negative ions, inhibit the oscillations, so that the reaction of the corona to pulse excitations becomes oscillatory 
damped or exponentially damped. Pure argon coronas, with mainly metastable atom feedback, show no oscillatory behaviour at all.

5) Free-running linear positive glow oscillations may or may not occur, according to whether the ionization region has a negative resistance, and the drift region resistance is large enough.

6) Nonlinear positive glow pulses are formed because a positive ion shell, once formed close to the anode, first decreases and then increases the corona ionization when it drifts toward the outer electrode. The first decrease quenches all ionization. The next ionization pulse may start when the first positive ion shell has drifted sufficiently far out to restore the corona reproduction factor, if initiatory electrons are to be found.

\section{Acknowledgements}

The present paper owes much to discussions with and experimental results from visitors, staff and students in the ELION laboratory in Trondheim: A and M Goldman (Laboratoire de Physique des Décharges, Gif-sur-Yvette, France); M Laan and K Kudu (Gas Discharge Laboratory, Tartu University, Estonia); B Kurdelova, M Kurdel and K Hensel (Comenius Unicersity, Bratislava, Slovakia); B Bjones, I H (Olsen) Lågstad, R T Randeberg, and G Løfsgård (ELION).

\section{References}

[1] Sigmond R S "Corona Discharges", Chapt. 4 in Meek J M and Craggs J D (eds) "Electrical Breakdown of Gases", (Wiley: London 1978) pp 319-84.

[2] Goldman M, Sigmond R S "Corona Discharges and Applications", in Kunhardt E E and Luessen L $\mathrm{H}$ (eds) "Electrical Breakdown and Discharges in Gases", NATO ASI Series B: Physics, Vol.89B (Plenum: New York 1983) pp 1-64.

[3] Loeb L B "Electrical Coronas", Univ. of California Press 1965

[4] Hermstein W, Archiv für Elektrotechnik 44 209-224 and 279-288 (1960)

[5] Uhlig C A E, Proc. High Voltage Symp., Nat. Res. Council Pub. No 4313 pp 15-1 - 15-13 and 16-1 16-11, Ottawa 1956

[6] Colli L, Facchini U, Gatti E, Persano A "Dynamics of corona discharge between cylindrical electrodes", J.Appl.Phys. 25 429-435 (1954)

[7] Beattie J "The Positive Glow Discharge”, Ph.D. thesis, Univ. of Waterloo, Canada 1975

[8] Linhjell D, Sigmond R S "Sampling image intensifier camera study of the glow pulses of positive point-to-plane coronas", Proc. $9^{\text {th }}$ Int. Conf. on Gas Discharges and Their Applications, Venezia (Benetton) 1988 pp 403-406

[9] Sigmond R S "Corona physics and diagnostics", Proc. 22. Int. Conf. on Phenomena in Ionized Gases, AIP Conf. Procs. 363, New York 1995 pp 332-344

[10] Kudu K, Lågstad I H, Sigmond R S "About the positive corona discharge forms in $\mathrm{O}_{2}-\mathrm{N}_{2}$ mixtures", Proc. 23. Int. Conf. on Phenomena in Ionized Gases, Toulouse 1997 pp IV-34 - 35

[11] These measurements were done in my Trondheim laboratory by students $\mathrm{K}$ Hensel from Comenius University, Bratislava, and I H Lågstad and T Randeberg, NTNU.

[12] Sigmond R S "GPCS1: A discharge model for all seasons", Proc. $8^{\text {th }}$ Int. Conf. on Electrostatics, Poitiers June 4-6 1977 (ed. G Touchard), Additional Issue pp 58-63. CIRCSIM with GPCS1 is free, available from the author (one 1.44 Mb diskette), email sigmond@phys.ntnu.no.

[13] Morrow R, personal communication "Positive glow corona theory", to be presented as topical invited lecture at $12^{\text {th }}$. Int. Conf. on Gas Discharges and Their Applications, Greifswald Sept. 1997.

[14] Sigmond R S "Basic corona phenomena" Proc. 16. Int. Conf. on Phenomena in Ionized Gases (Duesseldorf 1983) Invited Papers Volume pp 174-186.

[15] Brown S C "Basic Data of Plasma Physics" Wiley: New York 1959.

[16] Sigmond R S, "On the static and quasistatic characteristics of the self-sustained low pressure Townsend discharge in hydrogen", Proc. $5^{\text {th }}$ Int. Conf. on Phenomena in Ionized Gases (North-Holland), 1962, pp 1359-1363. 